Kuchnicka Aleksandra, Zielińska Martyna, Zarankiewicz Natalia, Kosz Katarzyna, Piecewicz-Szczęsna Halina. Risk factors of ovarian cancer: family history, obesity and oral contraceptive use. Journal of Education, Health and Sport. 2020;10(9):397-402. eISSN 2391-8306. DOI http://dx.doi.org/10.12775/JEHS.2020.10.09.046

https://apcz.umk.pl/czasopisma/index.php/JEHS/article/view/JEHS.2020.10.09.046

https://zenodo.org/record/4035636

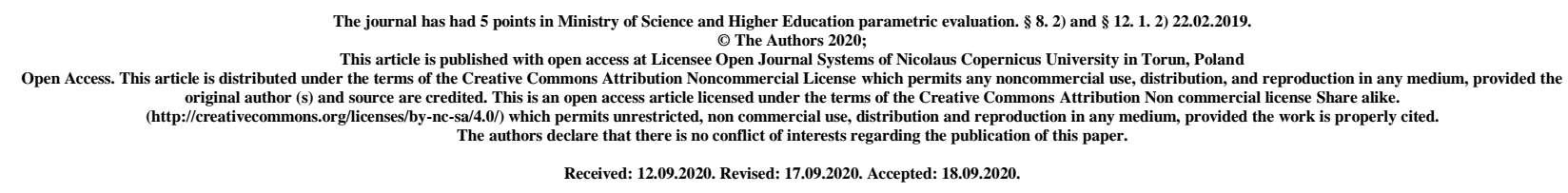

\title{
Risk factors of ovarian cancer: family history, obesity and oral contraceptive use
}

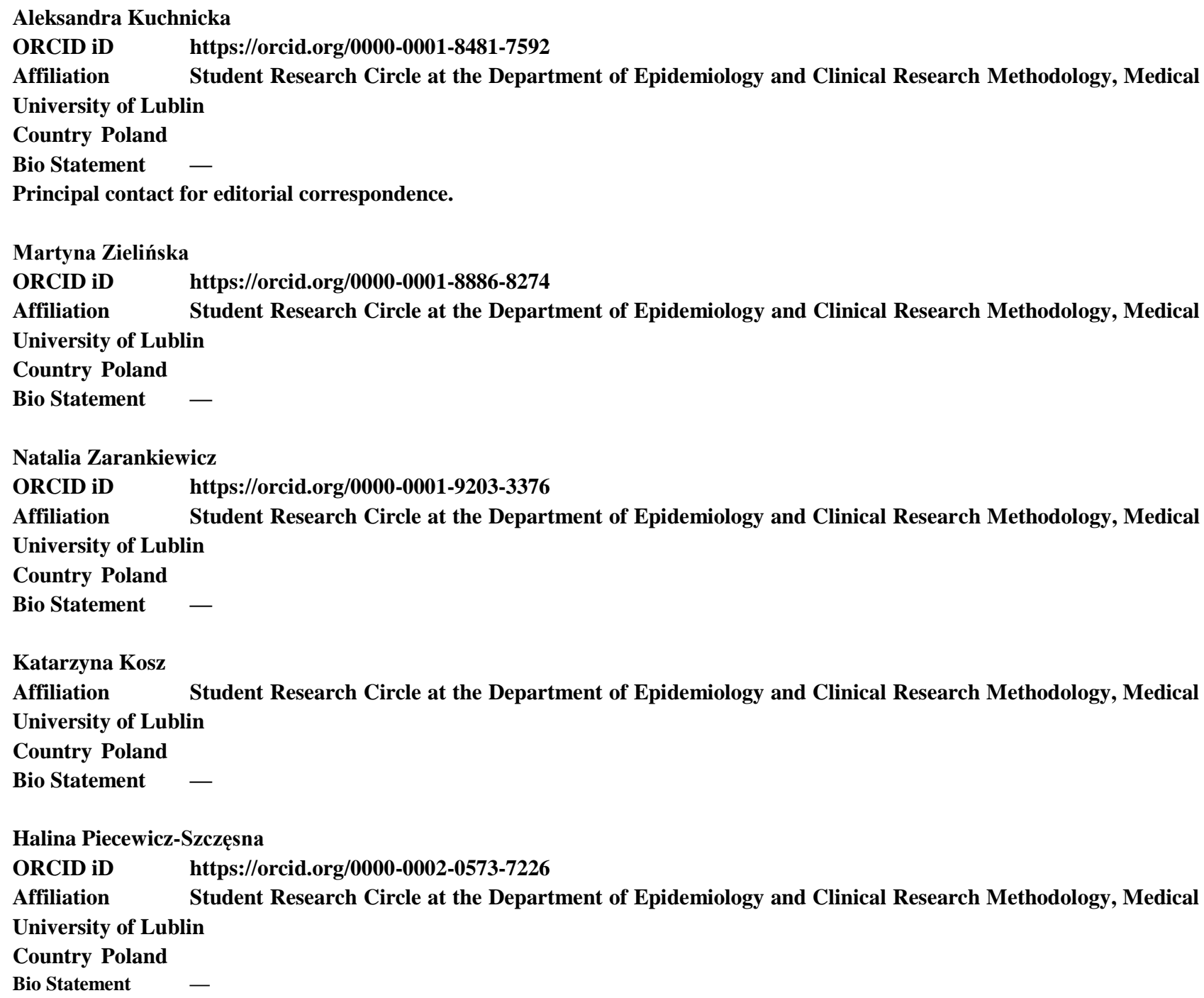




\begin{abstract}
Introduction and purpose:

Ovarian cancer (OC) takes the 7th place among most common female malignancies worldwide. There are almost 240 thousand of new cases diagnosed each year and the number is constantly growing. Defining risk factors of OC is essential to provide good education among women and predict this malignancy better. The aim of this review is to analyze the current data on risk factors of OC concentrating on family history, obesity and oral contraceptives use.
\end{abstract}

\title{
Material and method:
}

This review was based on articles from 2010 to 2020 collected in PubMed and concerning the impact of several risk factors on developing OC.

\section{Results:}

The best described risk factor of OC is genetics and family history. 75\% or hereditary OCs are associated to BRCA genes mutations, especially BRCA1. Other mutations increasing the risk of OC are MSH2 and MLH1 genes mutations. Diverse risk factors correlate with different histological subtypes of OC. Obesity increases the levels of estrogens, androgens and leptin, which may promote the proliferation of ovarian epithelial cells. Using oral contraceptives is related to $20 \%$ decreased risk of OC for each 5 years of use.

\section{Conclusions:}

BRCA1/2, MSH2 and MLH1 mutations and obesity are well-defined risk factors of ovarian cancer. The first-choice risk-reducing procedure among BRCA carriers is bilateral salpingo-oophorectomy. On the contrary, using oral contraceptives results in decreased risk of OC. Promoting knowledge about OC risk factors among women and healthcare professionals is crucial in order to reduce the number of ovarian cancer.

Key words: ovarian cancer; risk factors; obesity; oral contraceptive; BRCA; family history

\section{Introduction and purpose}

Ovarian cancer (OC) is caused by an incorrect and unceasing growth of the neoplastic cells derived from ovarian cells. It forms one of most frequent fatal gynecologic disorders which states the seventh leading cancer diagnosis among women in the world population [1,2]. According to the worldwide data, there are around 239,000 new cases and it causes 152,000 deaths each year [2]. The major problem is that women make appointments with medical experts definitely too late, and most of the cases are diagnosed with advanced stage of the cancer. It is certainly connected with the fact that patients are taking no notice of the symptoms. [3] Moreover, the 5 years survival rate in the III, IV staging of the disease can be measure up to $25 \%$ [2]. Each year the data about increasing number of cases begs the question of how to provide better prediction of those [4].

OC may be labeled as a diverse group of diseases. The crucial role in understanding the risk factors connection plays the stratification grounded on subtypes. Looking into the pathological classification of OC, there are three types of cells that ovarian tumors have origins in: epithelial, stromal and germ cells. Compelling data show that the epithelial OC concern women above age 40 and occurs most often in developed countries (over 90\%). [2,4] Surprisingly, most malignant tumors seem to come from other gynecological tissues, involving the ovary secondarily [2]. 


\section{Description of the state of knowledge}

\section{Genetic risk factors}

According to the studies, the family history of $\mathrm{OC}$ is one of the most established risk factor of the disease. Consequently, women with at least one affected first-degree relative are having about three times higher risk that women with no affected relatives. That clearly shows the importance of the medical interview and genetic testing [4]. About $75 \%$ of all hereditary OC are caused by mutations in the BRCA genes, with the prevalence of autosomal dominant inheritance. Female BRCA1 mutation carriers have the lifetime risk for OC development estimated between 39-46\%, whereas the female BRCA2 mutation carriers around 11-27\%. Furthermore, studies have demonstrated that women with the BRCA2 mutation present the tendency to both breast and OC around 10 years later in comparison to BRCA1 female carriers [5]. Nonetheless, the high proportion of those mutations affects women without family history (either OC or breast). Both of those mutations are strongly connected with a high-grade serous tumor. Additionally, the hereditary nonpolyposis colorectal cancer (HNPCC) or the Lynch syndrome forms the meaningful OC risk factor. This condition is characterized by the defective DNA mismatch repair (MMR; MLH1, MSH2, MSH6, PMS2 genes), or the regulator of MSH2: epithelial cell adhesion molecule (EPCAM). The mutation of MSH2/EPCAM and MLH1 genes enhance the OC risk up to 24\% [5].

The high risk might be decreased by up to $80-90 \%$ by removing the ovarian and fallopian tubes [6]. Currently, the risk-reducing bilateral salpingo-oophorectomy ( $\mathrm{rBSO}$ ) is presenting prime strategy in decreasing the risk of OC in BRCA mutation carriers. This method is recommended for those women mutation carriers at age 35-40 or after completing the childbearing. Tschernichovsky et al., created a systematic review using the studies from 1995-2016. The metanalysis in 2009, collecting data from over 2,840 participants, show that rBSO reduced $80 \%$ of ovarian and fallopian tube cancer frequency in mBRCA carriers (comparing to those who had not undergone this procedure). [7] However, this protection might be contingent on the mutation type. The studies collected from more than 4000 patients by Marchetti et al., compared the data from the postprocedural hazard ratio (HR) in BRCA1 and BRCA2 carriers. Firstly, the conclusion was clear, that the risk-reducing salpingo-oophorectomy (RRSO) effectively decreases the risk over the control group. The performed analysis showed that the HR for OC was 0,20 for women carring the BRCA1 mutation, whereas the BRCA2 patients did not show the demonstrable benefit [8]. Moreover, the all-cause mortality benefit connected with RRSO was 0,32 .

Wentzensen et. al pooled a data from over 1,3 million women (21 studies) and demonstrated that most of the risk factors presents histological heterogeneity [9]. The Ovarian Cancer Cohort Consorium (OC3) evaluated the correlation between 14 key risk factors with the invasive epithelial OC risk overall and by the histologic subtype. The studies focused on factors such as: smoking, parity, oral contraceptive use, menopausal hormone therapy use and body mass index (BMI). According to this study, the parous contrarily to nulliparous women reduced morbidity rate of all OC subtypes (with the prevalence of clear cell carcinomas risk reduction) [9]. Furthermore, the association between the 5-year later menopause, the endometrioid and clear cell carcinomas was noted. In regard to menopausal hormone therapy use, the 5-year increase was noticeably connected with the increased risk of endometrioid and serous carcinomas and with decreased risk of clear cell carcinoma. As for the other connections, it has been proven that the family history was significantly associated with OC risk overall. More specifically, a first-degree family history is strongly factor into increased risk of serous tumors. 
Smoking factor was associated only with the mucinous carcinomas, and as for BMI it has been proven that it was not meaningfully connected with any particular subtype.

\section{Obesity and diet}

In 2014 World Cancer Research Fund/American Institute for Cancer Research marked a body fatness as a probable risk factor for OC. It might be explained by the fact, that the adipose tissue is an endocrine organ which performs functions such as: glucose homeostasis, immunity and more (apart from being a calorie storage). Obesity is a condition which presents the increased level of circulating estrogen and androgen, especially among postmenopausal women. The positive association between estrogens and OC risk may be explained by promoting the proliferation of ovarian epithelial cells [10]. The steroid hormones such as estrogen and progesterone are synthesized from cholesterol. Women with high fat diet are providing the substrates for the excessive estrogen synthesis, which consequently activates cell proliferation in genital tract [11]. Additionally, obese women may ovulate less frequently because of those obesity-related hormonal imbalances. They are more likely -in their peri-menopausal years- to undergo hysterectomy and bilateral salpingo-oophorectomy, either [12].

High lipid levels have impact upon the leptin - the hormone secreted by the adipose tissue. This hormone has several functions in body regulations such as: feeling of satiety or stimulation the release of gonadotropin-releasing hormone $(\mathrm{GnRH})$, which in the process stimulates release of luteinizing hormone $(\mathrm{LH})$ and follicle-stimulating hormone (FSH). Increased levels of LH may eventuate in immature release of the ovum. Further, the high saturated fat intake, high circulating cholesterol levels in the circulation system and consequently increased levels of estrogen may result in wrong re-epithalialisation of the ovaries [11].

In the publication from 2017, Foong et. al have examined the linkage between obesity and OC risk. The assessment was based on 43 studies and BMI was used as an exposure measure of obesity. A statistically positive association between OC risk and increased BMI were found in 14 studies. Contrarily, a negative association were found in 3 case-control studies. Furthermore, in 26 studies have been reported no statistically significant association [12]. Accordingly, the results from Collaborative Group on Epidemiological Studies of OC [13] show the interrelation grounded on data collected from 47 studies. The conclusion was clear: the more obese and heavier women were, the greater was the risk of OC. Interestingly, the risk of OC was higher with increasing BMI among never-users of menopausal hormonal therapy, however not among the ever-users.

The association between BMI, progression-free survival (PFS), ovarian cancer-specific survival and overall survival was analyzed based on data which included 21 studies and 12390 women with invasive OC. The results show that overweight, obese and morbidly obese women had experienced much worse survival in comparison to normal weight women. According to this study, the obesity was connected with 10-12\% OS disadvantage amongst women with OC (similar for PFD and ovarian cancer-specific mortality). As for the subtype analyses, the connection was strongest for women with endometrioid and low-grade serous cancers and lesser for high-grade serous cancers. [14]

As regards to treatment, obese women may have worse survival wherethrough to the practice of dose capping while prescribing the chemotherapy. Dose capping is involving the use of ideal body weight (rather than actual) when calculating the quantity to be given. Those practices are done because of the potential for chemotherapy-related toxicity when the full dose is given $[14,15]$. 


\section{Oral contraceptives}

The medical literature has consonantly apprised the association between the use of oral contraceptives and the risk of OC. The potential means for reducing the high genetic risk of developing OC is chemoprevention with oral contraceptives use [16]. In compliance with available publications, the protective effect increases with the longer period of use (about $20 \%$ lesser risk for each 5 years of use). Furthermore, reduction of the risk does not seem to be specific to any OC histotype or oral contraceptive formulation [2].

Iodice et al. in one retrospective cohort and four case-control studies collected data from 1262 cases with BRCA1 and 253 cases with BRCA2 mutation. In meta-analysis a risk reduction for OC was confirmed with the use of oral contraceptives. In conformity with the previously cited studies, the increasing duration of oral contraceptives use was connected with the linear decrease in risk of $36 \%$ (for each additional 10 years) [17].

The question about the association between BRCA1/2 mutation and reduction of the risk of OC was posed by the Moorman et al. [16] item. The assessment of four studies suggested a stronger inverse association among female patients with longer duration of oral contraceptive use, however the clear linear connections were not present in all of them. One study comparing women who were using oral contraceptive for less than 10 year or $\geq 10$ years ago with never users. The result presents that the risk was lower among more recent users (both for BRCA1 and BRCA2 mutation carriers).

\section{Summary}

The OC forms a severe condition which occurs more and more female patients. Considering that this disease is often detected by physicians in late stages, the regular check-ups are playing crucial role. Consequently, the knowledge about the risk factors should be widely spread to minimize the potential chance of developing a disease. It is essential to emphasize the protective impact of using oral contraceptives and the importance of reducing obesity among women. The prime strategy reducing the risk among BRCA genes mutations carriers is bilateral salpingooophorectomy, which should be considered individually. 


\section{References}

1. Dinca AL, Bîrla RD, Dinca VG, Marica C, Panaitescu E, Constantinoiu S. Prognostic Factors in Advanced Ovarian Cancer - A Clinical Trial. Chirurgia (Bucur). 2020;115(1):50-62. doi: 10.21614/chirurgia.115.1.50

2. Reid, B. M., Permuth, J. B., \& Sellers, T. A. (2017). Epidemiology of ovarian cancer: a review. Cancer biology \& medicine, 14(1), 9-32. https://doi.org/10.20892/j.issn.2095-3941.2016.0084

3. Mallen, A. R., Townsend, M. K., \& Tworoger, S. S. (2018). Risk Factors for Ovarian Carcinoma. Hematology/Oncology Clinics of North America. doi:10.1016/j.hoc.2018.07.002

4. Webb PM, Jordan SJ. Epidemiology of epithelial ovarian cancer. Best Pract Res Clin Obstet Gynaecol. 2017;41:3-14. doi:10.1016/j.bpobgyn.2016.08.006

5. Kari L. Ring, Christine Garcia, Martha H. Thomas, Susan C. Modesitt, Current and future role of genetic screening in gynecologic malignancies, American Journal of Obstetrics and Gynecology, Volume 217, Issue 5, 2017, Pages 512-521, https://doi.org/10.1016/j.ajog.2017.04.011.

6. Adrianne R. Mallen, Mary K. Townsend, Shelley S. Tworoger, Risk Factors for Ovarian Carcinoma, Hematology/Oncology Clinics of North America, Volume 32, Issue 6, 2018, Pages 891-902, https://doi.org/10.1016/j.hoc.2018.07.002.

7. Tschernichovsky R, Goodman A. Risk-Reducing Strategies for Ovarian Cancer in BRCA Mutation Carriers: A Balancing Act. Oncologist. 2017;22(4):450-459. doi:10.1634/theoncologist.2016-0444

8. Marchetti C, De Felice F, Palaia I, et al. Risk-reducing salpingo-oophorectomy: a meta-analysis on impact on ovarian cancer risk and all cause mortality in BRCA 1 and BRCA 2 mutation carriers. BMC Womens Health. 2014;14:150. Published 2014 Dec 12. doi:10.1186/s12905-014-0150-5

9. Wentzensen N, Poole EM, Trabert B, et al. Ovarian Cancer Risk Factors by Histologic Subtype: An Analysis From the Ovarian Cancer Cohort Consortium. J Clin Oncol. 2016;34(24):2888-2898. doi:10.1200/JCO.2016.66.8178

10. Tworoger SS, Huang T. Obesity and Ovarian Cancer. Recent Results Cancer Res. 2016;208:155-176. doi:10.1007/978-3-319-42542-9_9

11. Dunneram Y, Greenwood DC, Cade JE. Diet, menopause and the risk of ovarian, endometrial and breast cancer. Proc Nutr Soc. 2019;78(3):438-448. doi:10.1017/S0029665118002884

12. Foong KW, Bolton H. Obesity and ovarian cancer risk: A systematic review. Post Reprod Health. 2017;23(4):183-198. doi:10.1177/2053369117709225

13. Collaborative Group on Epidemiological Studies of Ovarian Cancer (2012) Ovarian Cancer and Body Size: Individual Participant Meta-Analysis Including 25,157 Women with Ovarian Cancer from 47 Epidemiological Studies. PLOS Medicine 9(4): e1001200. https://doi.org/10.1371/journal.pmed.1001200

14. Nagle CM, Dixon SC, Jensen A, et al. Obesity and survival among women with ovarian cancer: results from the Ovarian Cancer Association Consortium. Br J Cancer. 2015;113(5):817-826. doi:10.1038/bjc.2015.245

15. Au-Yeung G, Webb PM, DeFazio A, Fereday S, Bressel M, Mileshkin L. Impact of obesity on chemotherapy dosing for women with advanced stage serous ovarian cancer in the Australian Ovarian Cancer Study (AOCS). Gynecol Oncol. 2014;133(1):16-22. doi:10.1016/j.ygyno.2014.01.030

16. Moorman PG, Havrilesky LJ, Gierisch JM, et al. Oral contraceptives and risk of ovarian cancer and breast cancer among high-risk women: a systematic review and meta-analysis. J Clin Oncol. 2013;31(33):4188-4198. doi:10.1200/JCO.2013.48.9021

17. Iodice S, Barile M, Rotmensz N, Feroce I, Bonanni B, Radice P et al (2010) Oral contraceptive use and breast or ovarian cancer risk in BRCA1/2 carriers: a meta-analysis. Eur J Cancer 46(12):2275-2284

18. Iversen L, Sivasubramaniam S, Lee AJ, Fielding S, Hannaford PC. Lifetime cancer risk and combined oral contraceptives: the Royal College of General Practitioners' Oral Contraception Study. Am J Obstet Gynecol. 2017;216(6):580.e1-580.e9. doi:10.1016/j.ajog.2017.02.002 